\title{
Electron acceleration by the reconnection outflow shock during solar flares
}

\author{
G. Mann, H. Aurass, and A. Warmuth
}

\author{
Astrophysical Institute Potsdam (AIP), An der Sternwarte 16, 14482 Potsdam, Germany \\ e-mail: gmann@aip.de
}

Received 9 February 2006 / Accepted 27 March 2006

\begin{abstract}
During solar flares a large amount of nonthermal electromagnetic radiation up to the $\gamma$-ray range is emitted from the corona, which implies that energetic electrons are generated. Within the framework of the magnetic reconnection scenario, jets appear in the outflow region and can establish standing fast-mode shocks if they penetrate the surrounding plasma at super-Alfvénic speed. These shocks can be a source of energetic electrons. During the solar event on October 28, 2003, an enhanced flux of hard X-and $\gamma$-rays up to $10 \mathrm{MeV}$ as observed by the INTEGRAL spacecraft indicates the generation of relativistic electrons. The radio signature of a standing shock wave appeared simultaneously with the enhanced hard X-and $\gamma$-ray fluxes. Here, we assume this shock is the source of the highly energetic electrons needed for the hard X-and $\gamma$-ray, as well as for the nonthermal radio radiation. The electrons are energized by shock drift acceleration, which is treated in a fully relativistic manner. After acceleration, the electrons travel along the magnetic field lines towards the denser chromosphere, where they emit hard X-and $\gamma$-ray radiation via bremsstrahlung. The observed photon fluxes in the range 7.5-10 MeV are explained by these theoretical results that adopt the coronal conditions found for the event on October 28, 2003.
\end{abstract}

Key words. accelerataion of particles - shock waves - Sun: flares - Sun: X-rays - Sun: radio radiation

\section{Introduction}

In October/November 2003 several flares of X-class importance were observed. The event on October 28, 2003 especially showed a strong enhancement of the hard X-and $\gamma$-ray radiation observed by the INTEGRAL (Gros et al. 2004) and RHESSI (Lin et al. 2002) spacecrafts. Hard X- and $\gamma$-ray radiation is generated by relativistic electrons, which are initially accelerated by the flare process in the corona and, subsequently, travel along the magnetic field lines towards the denser chromosphere, where they emit electromagnetic radiation via bremsstrahlung (see e.g. Brown 1971, 1972). Thus, the appearance of hard Xand $\gamma$-ray radiation implies the generation of highly energetic electrons during the flare process.

If a prominence (EP) is destabilized and, subsequently, rises up, it stretches the underlying magnetic field lines (Fig. 1), which leads to the formation of a current sheet. If the current exceeds a critical value there, the resistivity is suddenly increased by plasma-wave excitation due to various instabilities (see e.g. Treumann \& Baumjohann 1997). Then, magnetic reconnection can take place in the region of enhanced resistivity (also called diffusion region, DR). Due to the strong curvature of the magnetic field lines in the vicinity of the diffusion region, the slowly inflowing plasma shoots away from the reconnection site as a hot jet (see Fig. 1). If the speed of these jets is super-Alfvénic, a fast magnetosonic shock, also called termination shock (TS) can be established due to the deceleration of the jet. The appearance of such shocks is predicted in the numerical simulations by Forbes (1986) and Shibata et al. (1995).

As well-known, only electric fields are able to accelerate electrons. An electric field is induced within the diffusion region, where electrons can be accelerated directly (Holman 1985; Benz 1987; Litvinenko 2000). Since the real phase space of the diffusion region is very small, Tsuneta \& Naito (1998) propose that the shock wave established in the outflow region of the reconnection site could be the source of the energetic electrons needed for the hard X-ray radiation during flares. Aurass et al. (2002) and Aurass \& Mann (2004) first reported the radio signatures of such shocks.

The solar event on October 28, 2003 has revealed that the hard X- and $\gamma$-ray fluxes are associated with the radio spectral signature of the TS during the impulsive phase of the flare (Sect. 2). Therefore, this shock is considered as the source of the energetic electrons needed for the hard X-and $\gamma$-ray emission. The generation of highly relativistic electrons via shock drift acceleration is investigated in Sect. 3. The theoretically obtained results are compared with the observations in Sect. 4.

\section{Observations}

On October 28, 2003 a huge X17 flare occurred in active regions NOAA 10486-10491 between 09:51 and 12:00 UT. The event became impulsive at 11:01 UT after a slow and intermittent rise in the release of energy. Gros et al. (2004), Share et al. (2004), Miroshnishenko et al. (2005), and Bieber et al. (2005) all discuss hard $\mathrm{X}$ - and $\gamma$-rays, as well as particle data, for this most intense solar energetic particle event of cycle 23 (Gopalswamy et al. 2005).

During a short time interval between 11:02:30 and 11:04:30 UT of the impulsive flare phase, the dynamic radio spectrum recorded by the radio spectralpolarimeter $(40-800 \mathrm{MHz})$ of the Astrophysical Institute Potsdam 


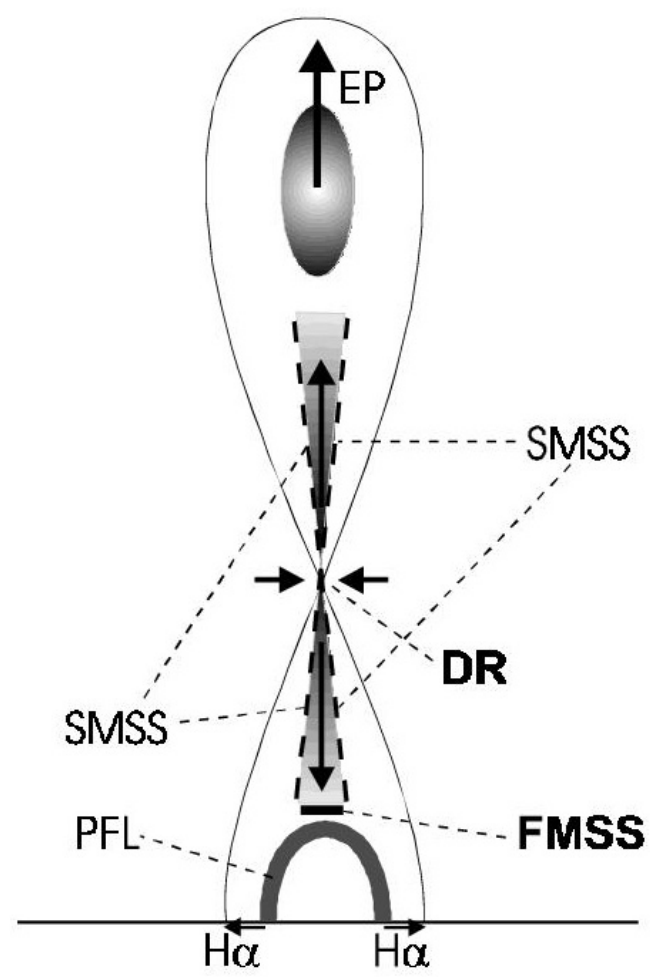

Fig. 1. Sketch of the generation of the termination shock (TS) at the flare (EP, erupting prominence; DR, diffusion region; SMSS, slowmode standing shock; FMSS, fast-mode standing shock also called termination shock, TS; PFL, post-flare loop). The grey shaded areas with the black arrows show the reconnection outflow jets.

(Mann et al. 1992) showed two harmonically related roughly non-drifting lanes in the frequency range between 100 and $500 \mathrm{MHz}$. They can doubtlessly be identified as a fundamental $(\mathrm{F})$ and harmonic $(\mathrm{H})$ type II burst-like emission, since they show the typical components of such bursts, i.e. the (non-drifting) "backbone" and the "herringbones" (HB) shooting away from the backbone towards both higher and lower frequencies (see e.g. Nelson \& Melrose 1985; and Mann 1995, for reviews). In two previous papers (Aurass et al. 2002; Aurass \& Mann 2004), it was shown that such spectral signatures can be interpreted as the radio signature of the TS that is formed in the lower and, possibly, also in the upper reconnection outflow jet during the flare (see Fig. 1).

Figure 2 shows the time derivative of the dynamic spectrum around this time interval. The frequency range encloses the five imaging frequencies (white arrows in Fig. 2) of the Nançay radio heliograph (NRH, Kerdraon et al. 1996). The spectral feature with the fast drifting HBs is regarded as the radio signature of the lower TS. This feature was identified for the first time in both harmonic modes. This reveals that the shock-related radio source acts at the $150 \mathrm{MHz}$ level in the corona.

The present case is exceptional because $\mathrm{X}$ - and $\gamma$-ray data (Gros et al. 2004) show a strong enhancement of photon fluxes up to the $10 \mathrm{MeV}$ range exactly during the appearance of the TS radio spectral features (Fig. 2). This proves that the TS radio signature occurs simultaneously with a very efficient energization of a large amount of electrons.

In Fig. 3, a composed map of radio imaging information is presented for 11:03:12 UT, i.e. in the time interval of the TS occurrence. The map is overplotted on a SOHO-MDI image (Scherrer et al. 1995) with isolines of the photospheric

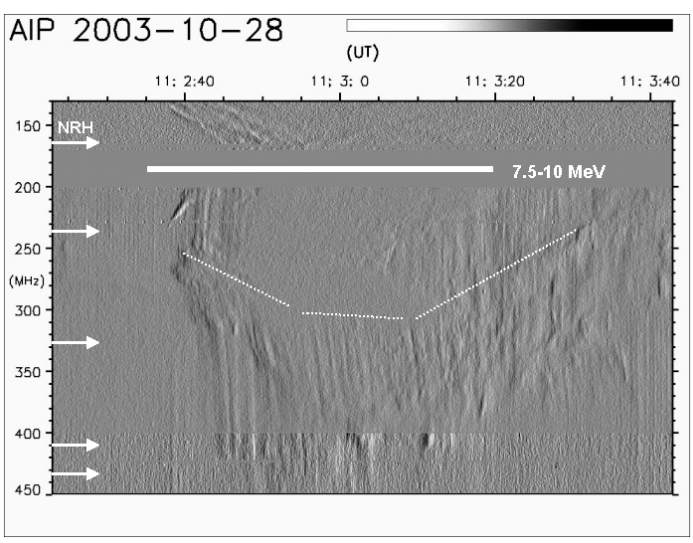

Fig. 2. The time-derived dynamic radio spectrum of the October 28, 2003 X-class flare shows the type II burst emission associated with the flare TS (gap between 170 and $200 \mathrm{MHz}$ due to local TV). Given is the frequency range of the Nançay radio heliograph (NRH, white arrows-the observing frequencies, comp. the imaging data in Fig. 3) and the time interval of significant enhancement of 7.5-10 MeV (white bar, after Gros et al. 2004). Fast drift features leaving the slightly curved backbone toward higher and lower frequencies are herringbone (HB) fine structures. Between 130 and $170 \mathrm{MHz}$ is the high frequency edge of F-mode. Above $200 \mathrm{MHz}$ is the $\mathrm{H}$-mode emission. The dashed white line roughly marks the onset of $\mathrm{HB}$ emission toward high frequencies. In the H-mode the backbone bandwidth is $<40 \mathrm{MHz}$ (upper limit of band splitting).

longitudinal magnetic field. The hard X-ray sources seen by RHESSI (Lin et al. 2002) are also shown as “+”. Unfortunately RHESSI only observed after 10:06 UT.

Just before the appearance of the TS in the dynamic radio spectrum, a radio source was situated above the active region NOAA 10486. After the onset of the TS (see Fig. 3), the corresponding radio source occurred far away from the flare ribbons. If seen over time, the source was firstly formed at $432-327 \mathrm{MHz}$, changed from simple to double-splitted at the highest frequencies of the H-mode, and also in the F-mode. The TS source occurred in projection about $0.3 R_{\odot}=210 \mathrm{Mm}$ away from chromospheric flare signatures and outside of the flaring active region but in the direction of one branch of the later visible CME. Note that the double-source structure visible at $432 \mathrm{MHz}$ in Fig. 3 fits well with rising, but inclined and somewhat twisted, post-flare loops above NOAA 10486 having footpoints near the RHESSI sources ("+" in Fig. 3, remind also Fig. 1).

Now, the TS is considered to be not only the source of the related type II burst-like radio waves but also of the energetic electrons that are generated by shock drift acceleration.

\section{Relativistic shock drift acceleration}

Fast magnetsonic shocks are accompanied by a density and magnetic-field compression (see e.g. Priest 1982). Consequently, they represent moving magnetic mirrors at which charged particles can be accelerated. Here, the TS is considered to be the source of energetic electrons generated via shock drift acceleration. Since it is intended to explain the production of highly energetic electrons $(\approx 10 \mathrm{MeV})$ as observed during the solar event on October 28, 2003, the shock drift acceleration must be treated in a fully relativistic manner (see e.g. Ball \& Melrose 2001, for the non-relativistic approach).

The shock wave is put in the $z-y$ plane and moves with speed $v_{\mathrm{s}}$ along the $x$ axis. The upstream magnetic field $\boldsymbol{B}_{\text {up }}$ is 


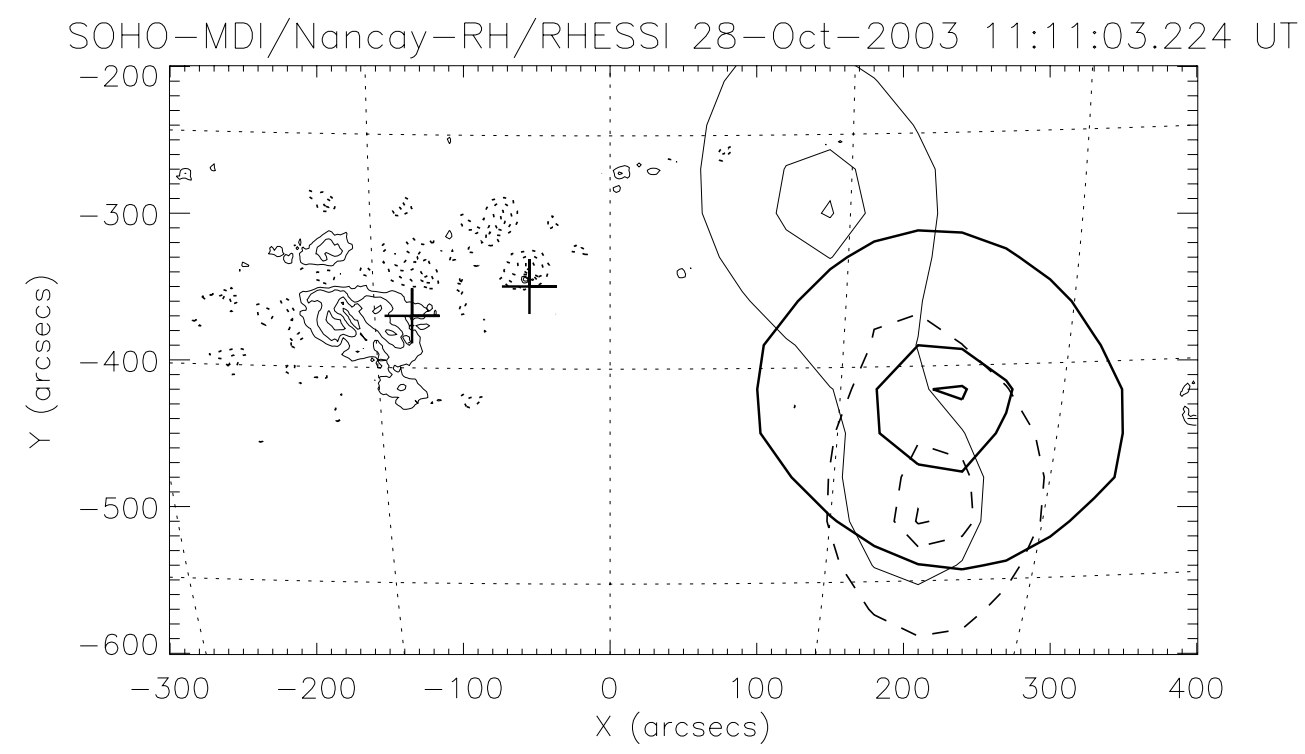

Fig. 3. Radio sources during termination shock emission, 11:03:12 UT (compare Fig. 2). For simplicity, we show only three of five NRH imaging frequencies that are harmonic mode TS emission: for NRH $432 \mathrm{MHz}$ (thin continuous), 410.5 (dashed), and $327 \mathrm{MHz}$ (thick continuous). The small-scale isolines, SOHO MDI 11:11:30 UT longitudinal field magnetogram, lines at .5, 1, 1.5 and $2 \times 10^{3}$ Gauss, reveal the dominant field structures of NOAA AR 10486. Large crosses (+) over the active region give the RHESSI hard X-ray source centroids 11:06-11:10 UT. The TS radio source is situated at least $0.3 R_{\odot}$ away from AR 10486, and 150 arcsec are taken for a characteristic equivalent half-power source diameter leading to an area of $A_{\text {source }}=2.9 \times 10^{20} \mathrm{~cm}^{2}$ as used in Sect. 4 .

located in the $z-x$ plane and takes an angle $\theta$ towards the $x$ axis, i.e. $\boldsymbol{B}_{\text {up }}=B_{\text {up }}(\cos \theta, 0, \sin \theta)$. Generally, shock drift acceleration represents a transformation of the particle velocities parallel and perpendicular to the magnetic field, i.e. $\left\{V_{\mathrm{i}, \|} ; V_{\mathrm{i}, \perp}\right\} \rightarrow\left\{V_{\mathrm{r}, \|} ; V_{\mathrm{r}, \perp}\right\}$. Here, the indices " $i$ " and " $r$ " denote the state before and after the reflection, respectively. The reflection process is usually performed in the de Hoffmann-Teller frame, where the shock is at rest and the motional electric field is removed, i.e. the upstream plasma flow is directed along the upstream magnetic field in this frame. Then, $v_{\mathrm{s}} \sec \theta$ is the shock speed in the de HoffmannTeller frame. According to the addition theorem of relativistic velocities (Landau \& Lifschitz 1982), the particle velocities in the de Hoffmann-Teller frame are related to the initial ones by

$\beta_{\mathrm{i}, \mathrm{HT}, \|}=\frac{\beta_{\mathrm{i}, \|}-\beta_{\mathrm{s}}}{1-\beta_{\mathrm{i}, \|} \beta_{\mathrm{s}}}$

and

$\beta_{\mathrm{i}, \mathrm{HT}, \perp}=\frac{\beta_{\mathrm{i}, \perp}}{1-\beta_{\mathrm{i}, \|} \beta_{\mathrm{s}}} \cdot \sqrt{1-\beta_{\mathrm{s}}^{2}}$

with $\beta_{\mathrm{i}, \|}=V_{\mathrm{i}, \|} / c, \beta_{\mathrm{i}, \perp}=V_{\mathrm{i}, \perp} / c$, and $\beta_{\mathrm{s}}=v_{\mathrm{s}} \sec \theta / c$. The reflection takes place under the conservation of the kinetic energy

$\beta_{\mathrm{HT}, \|}^{2}+\beta_{\mathrm{HT}, \perp}^{2}=$ const.

and the magnetic moment

$\frac{\beta_{\mathrm{HT}, \perp}^{2}}{B}=$ const

resulting in $\beta_{\mathrm{r}, \mathrm{HT}, \|}=-\beta_{\mathrm{i}, \mathrm{HT}, \|}$ and $\beta_{\mathrm{r}, \mathrm{HT}, \perp}=\beta_{\mathrm{i}, \mathrm{HT}, \perp}$. Here, $B$ denotes the magnitude of the magnetic field. The influence of the electrostatic potential across the shock can be neglected when relativistic particles are considered, since it is on the order of a few $k_{\mathrm{B}} T$ ( $k_{\mathrm{B}}$, Boltzmann's constant; $T$, temperature in the upstream region). It is given by

$e \phi_{\mathrm{HT}}=\frac{\gamma}{(\gamma-1)} \cdot k_{\mathrm{B}} T \cdot\left[\left(\frac{N_{\mathrm{down}}}{N_{\mathrm{up}}}\right)^{(\gamma-1)}-1\right]$

( $\gamma$, ratio of the specific heats) in the de Hoffmann-Teller frame (Goodrich \& Scudder 1984; Kunic et al. 1999). Here, $N_{\text {down }}$ and $N_{\text {up }}$ denote the particle number densities in the down- and upstream region, respectively. Because of $N_{\text {down }} / N_{\text {up }} \leq 4$ for fast magnetosonic shocks (see e.g. Priest 1982), Eq. (5) provides $e \phi_{\mathrm{HT}} \leq 3.8 k_{\mathrm{B}} T$ for $\gamma=5 / 3$.

After performing the reflection process and fulfilling of the conservation laws (see Eqs. (3) and (4)), the particle velocities are tranformed from the de Hoffmann-Teller frame back to the laboratory frame by

$\beta_{\mathrm{r}, \|}=\frac{\beta_{\mathrm{r}, \mathrm{HT}, \|}+\beta_{\mathrm{s}}}{1+\beta_{\mathrm{i}, \mathrm{HT}, \| \beta_{\mathrm{s}}}}$

and

$\beta_{\mathrm{r}, \perp}=\frac{\beta_{\mathrm{r}, \mathrm{HT}, \perp}}{1+\beta_{\mathrm{i}, \mathrm{HT}, \| \beta_{\mathrm{s}}}} \cdot \sqrt{1-\beta_{\mathrm{s}}^{2}}$.

Finally, the particle velocities after the reflection are related to those in the initial state by

$\beta_{\mathrm{r}, \|}=\frac{2 \beta_{\mathrm{s}}-\beta_{\mathrm{i}, \|}\left(1+\beta_{\mathrm{s}}^{2}\right)}{1-2 \beta_{\mathrm{i}, \|} \beta_{\mathrm{s}}+\beta_{\mathrm{s}}^{2}}$

and

$\beta_{\mathrm{r}, \perp}=\frac{\left(1-\beta_{\mathrm{s}}^{2}\right)}{1-2 \beta_{\mathrm{i}, \|} \beta_{\mathrm{s}}+\beta_{\mathrm{s}}^{2}} \cdot \beta_{\mathrm{i}, \perp}$

where Eqs. (1) and (2) are inserted into Eqs. (6) and (7). In the non-relativistic limit Eqs. (8) and (9) transfer into the wellknown ones, i.e. $V_{\mathrm{r}, \|}=2 v_{\mathrm{s}} \sec \theta-V_{\mathrm{i}, \|}$ and $V_{\mathrm{r}, \perp}=V_{\mathrm{i}, \perp}$ (Holman $\&$ Pesses 1982; Ball \& Melrose 2003). 


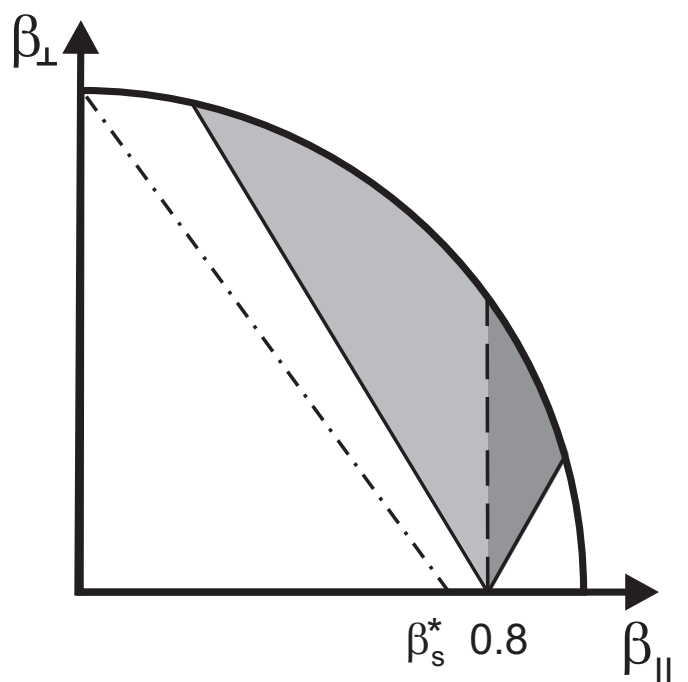

Fig. 4. Illustration of the shock drift acceleration in the $\beta_{\perp}-\beta_{\|}$plane. For a shock wave with $\beta_{\mathrm{s}}=0.8$ and $B_{\text {down }} / B_{\text {up }}=2$, all particles in the grey area are transferred into the dark area. In the same case, all particles located right from the dashed-dotted line will be transmitted into the downstream region at a second encounter (see further explanation in Sects. 3 and 4.)

Likewise, the reflection conditions

$\beta_{\mathrm{i}, \|} \leq \beta_{\mathrm{s}}$

and

$\beta_{\mathrm{i}, \perp} \geq \frac{\tan \alpha_{\mathrm{lc}}}{\sqrt{1-\beta_{\mathrm{s}}^{2}}} \cdot\left(\beta_{\mathrm{s}}-\beta_{\mathrm{i}, \|}\right)$

must be satisfied by the initial particles in order to be accelerated. The loss-cone angle is defined by $\alpha_{\mathrm{lc}}=\arcsin \left[\left(B_{\text {up }} / B_{\text {down }}\right)^{1 / 2}\right]$, where $B_{\text {down }}$ denotes the magnitude of the magnetic field in the downstream region.

Thus, the acceleration process basically represents a transformation in the $\beta_{\perp}-\beta_{\|}$plane as illustrated in Fig. 4. For a shock wave with $\beta_{\mathrm{s}}=0.8$ and $B_{\text {down }} / B_{\text {up }}=2$, i.e. $\alpha_{\mathrm{lc}}=45^{\circ}$, the initial particles located in the grey area left of the dashed line are transformed into the dark one to the right of it.

It should be emphasized that there is a critical shock speed $\beta_{\mathrm{s}}^{*}$. Only particles with an initial velocity $\beta_{\mathrm{i}, \|}>0$ will be accelerated by shock waves with $\beta_{\mathrm{s}}>\beta_{\mathrm{s}}^{*}=\cos \alpha_{\mathrm{lc}}$ (see also Fig. 4). The other particles moving towards the shock in the laboratory frame, i.e. $\beta_{\mathrm{i}, \|}<0$, will be transmitted totally into the downstream region.

\section{Discussion}

The radio image of TS is observed around $300 \mathrm{MHz}$ as the $\mathrm{H}$-mode emission revealed from the spectral radio data (Fig. 2). Therefore, the plasma parameters usually found at the $150 \mathrm{MHz}$ level in the corona will be chosen as the basic parameters for the present discussion. An electron number density of $N_{0}=$ $2.8 \times 10^{8} \mathrm{~cm}^{-3}$ is related to this frequency. According to a twofold Newkirk (1961) coronal density model, the $150 \mathrm{MHz}$ level is located about $160 \mathrm{Mm}$ above the photosphere. There, a magnetic field strength of $4.7 \mathrm{G}$ can be expected above active regions (Dulk \& McLean 1978). Furthermore, a typical flare temperature of $10^{7} \mathrm{~K}$ is assumed for this discussion. All these parameters result in a thermal electron velocity $v_{\mathrm{th}, \mathrm{e}}=$ $12300 \mathrm{~km} \mathrm{~s}^{-1}$ and an Alfvén speed $v_{\mathrm{A}}=610 \mathrm{~km} \mathrm{~s}^{-1}$.

For the TS a jump of the density, $N_{\text {down }} / N_{\text {up }}=2$ (Aurass et al. 2002) is assumed because of the bandwidth of the nondrifting type II burst (Fig. 3). According to the RankineHugoniot relationships (see e.g. Priest 1982), it leads to a magnetic field compression $B_{\text {down }} / B_{\text {up }}=2$ across the shock and an Alfvén-Mach number $M_{\mathrm{A}}=2.3$, i.e. the shock speed is $v_{\mathrm{s}}=1500 \mathrm{~km} \mathrm{~s}^{-1}$. From the radio images, an area $A_{\text {source }}=$ $2.9 \times 10^{20} \mathrm{~cm}^{2}$ is derived for the TS-associated radio source (Fig. 3). Consequently, the total electron flux through the shock is found to be $P_{\mathrm{e}}=N_{0} \cdot A_{\text {source }} \cdot v_{\mathrm{A}} \cdot M_{\mathrm{A}}=1.1 \times 10^{37} \mathrm{~s}^{-1}$.

In order to determine the phase space density for the accelerated electrons, a velocity distribution function of electrons in the upstream region has to be introduced. In-situ spacecraft measurements (Lin 1974) have shown that electron distribution functions are usually not Maxwellian ones but have an enhanced supra-thermal tail in space plasmas. Thus, a kappa distribution defined by

$f=C_{\kappa} \cdot\left[1+\frac{E}{\kappa E_{\kappa}}\right]^{-\kappa-1}$

with the kinetic energy $E=m_{0} c^{2}\left[\left(1-\beta^{2}\right)^{-1 / 2}-1\right]$ (see e.g. Maksimovich et al. 1997; and Pierrard et al. 1999) is used for the initial velocity distribution for electrons in the upstream region for further discussions. Note that for energies $E \approx E_{K}$ and $E \gg E_{\kappa}$ the kappa distribution behaves like a Maxwellian and a power-law one, respectively. In the limit $\kappa \rightarrow \infty$, it totally tranforms into a Maxwellian distribution. Here, the distribution function is normalized to unity by fixing the constant $C_{K}$; i.e.

$C_{\kappa}^{-1}=4 \pi c^{3} \int_{0}^{\infty} \mathrm{d} \epsilon \cdot \frac{\sqrt{\epsilon(2+\epsilon)}}{(1+\epsilon)^{4}} \cdot\left[1+\frac{\epsilon}{\kappa \epsilon_{\kappa}}\right]^{-\kappa-1}$

with $\epsilon=E / m_{0} c^{2}$ and $\epsilon_{K}=E_{K} / m_{0} c^{2}$. According to the kinetic definition of the temperature, $\bar{E}=3 N_{0} k_{\mathrm{B}} T / 2$ or

$$
\begin{aligned}
\bar{\epsilon} & =\frac{\bar{E}}{m_{0} c^{2}}=\frac{3}{2} \cdot \frac{k_{\mathrm{B}} T}{m_{0} c^{2}} \\
& =4 \pi C_{\kappa} c^{3} \cdot \int_{0}^{\infty} \mathrm{d} \epsilon \cdot \epsilon \cdot \frac{\sqrt{\epsilon(2+\epsilon)}}{(1+\epsilon)^{4}}\left[1+\frac{\epsilon}{\kappa \epsilon_{\kappa}}\right]^{-\kappa-1}
\end{aligned}
$$

the parameter $\epsilon_{\kappa}$ is related to the temperature $T$ by

$\epsilon_{\kappa}=\frac{(\kappa-3 / 2)}{\kappa} \cdot \frac{k_{\mathrm{B}} T}{m_{0} c^{2}}$

After introducing the pitch angle $\vartheta$ according to $\beta_{\|}=\beta \cdot \cos \vartheta$ the number of electrons $\mathrm{d} N$ in the range $[\epsilon+\mathrm{d} \epsilon, \epsilon]$ and $[\vartheta+\mathrm{d} \vartheta, \vartheta]$ can be given by

$$
\frac{1}{N_{0}} \cdot \frac{\mathrm{d} N}{\mathrm{~d} \vartheta \mathrm{d} \epsilon}=2 \pi C_{\kappa} c^{3} \sin \vartheta \cdot \frac{\sqrt{\epsilon(2+\epsilon)}}{(1+\epsilon)^{4}} \cdot\left[1+\frac{\epsilon}{\kappa \epsilon_{\kappa}}\right]^{-\kappa-1}
$$

Figure 5 shows the energy dependence of $N_{0}^{-1} \cdot \mathrm{d} N / \mathrm{d} \epsilon$ for a temperature of $10^{7} \mathrm{~K}$ and $\kappa=2.5,10$, and $\infty$ after integrating with respect to the pitch angle $\vartheta$. As already mentioned, the case with $\kappa=\infty$ corresponds to a Maxwellian distribution. A value $\kappa=10$ seems to be appropriate in the solar corona (Maksimovich et al. 1997; Pierrard et al. 1999).

In order to illustrate the generation of relativistic electrons by the proposed mechanism the production of $8.54 \mathrm{MeV}$ $(\epsilon=16.68)$ electrons is demonstrated as an example. Note 


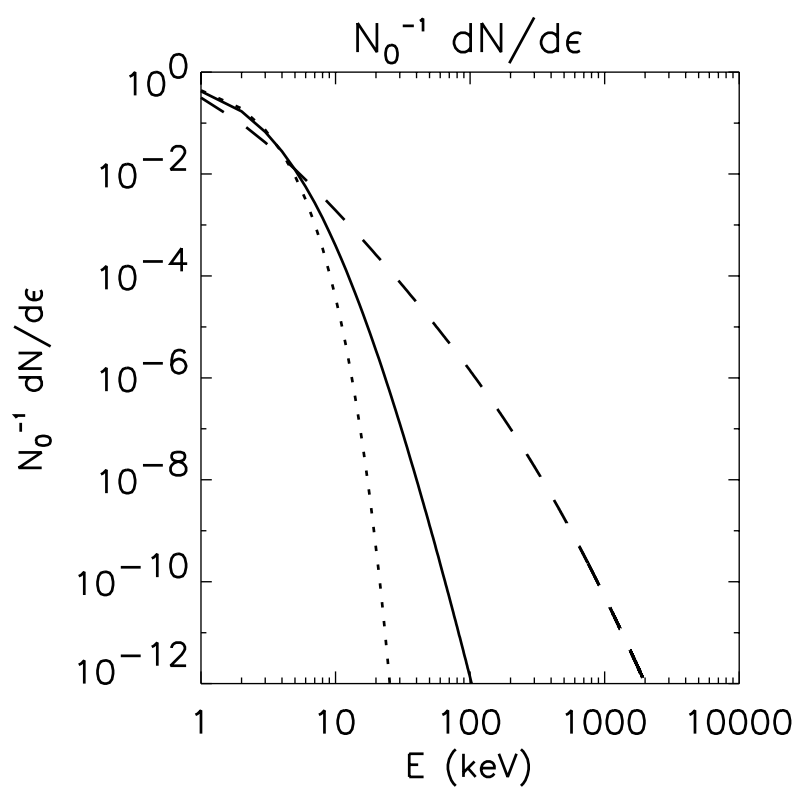

Fig. 5. The dependence of the function $N^{-1} \cdot \mathrm{d} N / \mathrm{d} \epsilon$ on the energy $E$ given in $\mathrm{keV}$ for a kappa distribution with $\kappa=2.5$ (dashed line), 10 (full line), and $\infty$ (dotted line). Note that $\kappa=\infty$ corresponds to a Maxwellian distribution.

that electrons of such an energy are needed for the emission of hard X-and $\gamma$-ray radiation up to $10 \mathrm{MeV}$, as observed by the INTEGRAL spacecraft during the event on October 28, 2003. An electron with an initial energy of $2.57 \mathrm{MeV}(\epsilon=5.03)$ and an inital pitch angle of $6.74^{\circ}$ (i.e. $\beta_{\mathrm{i}, \|}=0.9793$ and $\beta_{\mathrm{i}, \perp}=0.1157$ ) is accelerated by a shock wave with a speed of $1500 \mathrm{~km} \mathrm{~s}^{-1}$ and an angle $\theta=89.7115^{\circ}\left(\beta_{\mathrm{s}}=0.993\right)$ to a final state with an energy of $8.54 \mathrm{MeV}$ and a pitch angle of $2.25^{\circ}$ (i.e. $\beta_{\mathrm{r}, \|}=0.9976$ and $\beta_{\mathrm{r}, \perp}=0.0393$ ) via shock drift acceleration. Now, the number of $8.54 \mathrm{MeV}$ electrons generated by this mechanism has been determined. As previously demonstrated, such electrons are produced from electrons with an initial energy of $2.57 \mathrm{MeV}$ and an initial pitch angle of $6.74^{\circ}$. The number of such electrons is $N_{0}^{-1} \cdot \mathrm{d} N / \mathrm{d} \vartheta \mathrm{d} \epsilon=3.6 \times 10^{-28}$ (see Eq. (16)). For comparison, the number of electrons with an energy of $8.54 \mathrm{MeV}$ and a pitch angle of $2.25^{\circ}$ is found to be $N_{0}^{-1} \cdot \mathrm{d} N / \mathrm{d} \vartheta \mathrm{d} \epsilon=2.4 \times 10^{-34}$ in the upstream region according to Eq. (16) if no acceleration happens. Thus, the proposed acceleration mechanism enhances the number of $8.5 \mathrm{MeV}$ electrons by a factor of $1.5 \times 10^{6}$ in comparison to the state without any acceleration. Since the total flux of electrons through the shock is $1.1 \times 10^{37} \mathrm{~s}^{-1}, 4 \times 10^{11}$ electrons with an energy of $8.5 \mathrm{MeV}$ are produced per second. That impressively demonstrates the efficiency of the proposed mechanism.

After the acceleration at the TS, the electrons are reflected back towards the upstream region. Because of the curvature of the magnetic field lines in the outflow region of the reconnection site, the energized electrons will encounter the TS once more. Since the shock speed $\beta_{\mathrm{s}}=0.993>\beta_{\mathrm{s}}^{*}=0.707$ for $\alpha_{\mathrm{lc}}=45^{\circ}$ because of $B_{\text {down }} / B_{\text {up }}=2$, all electrons accelerated at the first shock encounter are totally transmitted into the downstream region at the second encounter. Then, they travel along the magnetic field lines towards the denser chromosphere, where they can emit hard X-and $\gamma$-ray radiation via bremsstrahlung.

\section{Summary}

During solar flares a large amount of electromagnetic radiation from the radio up to the hard X-and $\gamma$-ray range is emitted from the corona. In particular, the huge solar event on October 28, 2003 was accompanied by a strongly enhanced emission of hard $\mathrm{X}$ - and $\gamma$-ray radiation (up to $\sim 10 \mathrm{MeV}$ ) during the solar flare impulsive phase. Magnetic reconnection is one possible process for explaining a sudden energy release in the solar corona during flares. A jet of hot plasma streams away from the reconnection site. If the speed of this flow is super-Alfvénic, a shock wave (TS) can be established in the outflow region. The solar radio data showed signatures of such a shock only during the impulsive phase of this special event. The simultaneous appearance of enhanced photon fluxes up to $10 \mathrm{MeV}$ and the radio signatures of the TS (see Sect. 2) implies that the TS is the source of those electrons that generate hard X-and $\gamma$-rays.

Here, the highly energetic electrons are considered to be generated by shock drift acceleration at the TS. That requires generalizing shock drift acceleration in a fully relativistic manner. Electrons with energies of $\sim 10 \mathrm{MeV}$ can only be produced by a relativistic shock, which is connected to a nearly perpendicular shock geometry under coronal circumstances. With the adoption of the plasma parameters found in the corona during the event on October 28,2003, it has been demonstrated that the proposed mechanism is able to provide the enhanced flux of $8.5 \mathrm{MeV}$ electrons required for the emission of hard X-and $\gamma$-ray radiation as observed by the INTEGRAL spacecraft. They are produced from electrons with an initial energy of about $2.5 \mathrm{MeV}$. If one had chosen a Maxwellian distribution with a temperature of $10^{7} \mathrm{~K}$ for the electrons, significantly less than one $2.5 \mathrm{MeV}$ electron would enter the shock area $A_{\text {source }}$ per second. That demonstrates the necessity of choosing a kappa distribution, which is much more approriate for space plasmas than a Maxwellian one (Lin et al. 1996; Vocks \& Mann 2003).

The relativistic electrons produced at the TS can be the source of the nonthermal hard X-and $\gamma$-ray radiation in the chromospheric footpoints, as well as at the loop-top areas. Here, nonthermal radiation means that it is caused by electrons that do not fulfill a thermal (e.g. Maxwellian or undisturbed kappa) distribution. Note that the proposed mechanism would work for energetic protons $(<10 \mathrm{GeV})$, too, since shock drift acceleration is basically independent of the charge of the particles.

We have shown that the generation of highly energetic electrons by the TS is able to explain the enhanced photon fluxes up to $10 \mathrm{MeV}$, as observed by the INTEGRAL spacecraft during the solar event on October 28, 2003. This comprehensive observational and theoretical study therefore confirms the idea of Tsuneta \& Naito (1998) in a quantitative manner.

Acknowledgements. The authors thank G. Emslie, G. Holman, H. Hudson, R. P. Lin, and L. Vlahos for stimulating discussions during the 5th RHESSI Workshop in Locarno in June 2005. We gratefully acknowledge the use of the SOHO-MDI, RHESSI, and Nançay Multifrequency Radioheliograph data. The work was financially supported by the German space agency Deutsches Zentrum für Luft- und Raumfahrt (DLR), under grant No. 50 QL 0001.

\section{References}

Aurass, H., \& Mann, G. 2004, ApJ, 615, 526

Aurass, H., Vršnak, B., \& Mann, G. 2002, A\&A, 384, 273

Ball, L., \& Melrose, D. B. 2001, Publ. Astron. Soc. Austr., 18, 361

Benz, A. O. 1987, Sol. Phys., 111, 1

Bieber, J. W., Clem, J., Evenson, P., et al. 2005, Geophys. Res. Lett., 32, 302 
Brown, J. C. 1971, Sol. Phys., 18, 489

Brown, J. C. 1972, Sol. Phys., 26, 441

Dulk, G. A., \& McLean, D. J. 1978, Sol. Phys., 57, 235

Forbes, T. G. 1986, ApJ, 305, 553

Goodrich, C. C., \& Scudder, J. 1984, J. Geophys. Res., 89, 6654

Gopalswamy, N., Barbieri, L., Lu, G., Plunkett, S. P., \& Skoug, R. M. 2005, Geophys. Res. Lett., 32, 304

Gros, M., et al. 2004, in Proc. 5th INTEGRAL Workshop, ed. V. Schönfelder, et al., ESA-SP, 552, 669

Holman, G. D. 1985, ApJ, 293, 584

Holman, G. D., \& Pesses, M. E. 1983, ApJ, 267, 837

Kunic, Z., Cairns, I. H., \& Knock, S. A. 2002, J. Geophys. Res., 107, 10029

Landau, L. D., \& Lifshitz, E. M. 1975, The Classical Field Theory (Tarrytown N.Y.: Pergamon Press), 264

Lin, R. P. 1974, Sp. Sec. Rev., 16, 189

Lin, R. P., et al. 1996, Geophys. Res. Lett., 94, 1211

Lin, R. P., et al. 2002, Sol. Phys., 210, 3

Litvinenko, Y. E. 2000, in High Energy Solar Physics - Anticipating HESSI, ed. R. Ramaty, \& N. Mandzhavidze, ASPC Conf. Ser., 206, 167

Maksimovic, M., Pierrard, V., \& Lemaire, J. 1997, A\&A, 324, 725
Mann, G. 1995, in Coronal magnetic Energy Release, ed. A. O. Benz, \& A. Krüger, Lecture Notes in Physics (Heidelberg: Springer Verlag), 183

Mann, G., Aurass, H., Voigt, W., \& Paschke, J. 1992, in Proc. 1st SOHO Workshop, ESA-SP 348, 129

Miroshnichenko, L. I., et al. 2005, J. Geophys. Res., 110, 101029

Nelson, G. S., \& Melrose, D. 1985, Type II Bursts, in Solar Radiophysics, ed. D. J. McLean, \& N. R. Labrum (Cambridge: Cambridge University Press), 333

Newkirk, G. A. 1961, ApJ, 133, 983

Pierrard, V., Maksimovic, M., \& Lemaire, J. 1999, J. Geophys. Res., 104, 17021 Priest, E. R. 1982, Solar Magnetohydrodynamics (Dordrecht: Reidel Publ. Comp.)

Scherrer, P. H., Bogart, R. S., Bush, R. I., et al. 1995, Sol. Phys., 162, 129

Share, G., Murphy, R. J., Smith, D. M., \& Schwartz, R. A. 2004, ApJ, 615, L169

Shibata, K., Masuda, S., Shimojo, M., et al. 1995, ApJ, 451, L83

Treumann, R. A., \& Baumjohann, W. 1997, Adv. Space Plasma Phys. (London: Imperial College Press), 143

Tsuneta, S., \& Naito, T. 1998, ApJ, 495, L67

Vocks, C., \& Mann, G. 2003, ApJ, 593, 1134 